\title{
Characterization of a photonic strain sensor in silicon-on-insulator technology
}

\author{
Wouter J. Westerveld, ${ }^{1,2, *}$ Jose Pozo, ${ }^{2}$ Peter J. Harmsma, ${ }^{2}$ Ruud Schmits, ${ }^{2}$ Erik Tabak, ${ }^{2}$ Teun C. van den Dool, ${ }^{2}$ \\ Suzanne M. Leinders, ${ }^{3}$ Koen W.A. van Dongen, ${ }^{2}$ H. Paul Urbach, ${ }^{1}$ and Mirvais Yousefi ${ }^{2}$ \\ ${ }^{1}$ Optics Research Group, Faculty of Applied Sciences, Delft University of Technology, Lorentzweg 1, 2628 CJ Delft, The Netherlands \\ ${ }^{2}$ TNO, Stieltjesweg 1, 2628 CK Delft, The Netherlands \\ ${ }^{3}$ Laboratory of Acoustical Imaging and Sound Control, Faculty of Applied Sciences, \\ Delft University of Technology, Lorentzweg 1, 2628 CJ Delft, The Netherlands \\ ${ }^{*}$ Corresponding author: w.j.westerveld@tudelft.nl
}

Received November 21, 2011; revised December 19, 2011; accepted December 19, 2011; posted December 19, 2011 (Doc. ID 158465); published February 6, 2012

\begin{abstract}
Recently there has been growing interest in sensing by means of optical microring resonators in photonic integrated circuits that are fabricated in silicon-on-insulator (SOI) technology. Taillaert et al. [Proc. SPIE 6619, 661914 (2007)] proposed the use of a silicon-waveguide-based ring resonator as a strain gauge. However, the strong lateral confinement of the light in SOI waveguides and its corresponding modal dispersion where not taken into account. We present a theoretical understanding, as well as experimental results, of strain applied on waveguide-based microresonators, and find that the following effects play important roles: elongation of the racetrack length, modal dispersion of the waveguide, and the strain-induced change in effective refractive index. (C) 2012 Optical Society of America

OCIS codes: $120.4880,160.1050,130.7408,280.4788,160.6000,000.2190$.
\end{abstract}

Piezoresistive electronic strain gauges are frequently used in micromachined electromechanical systems (MEMS) [1]. Alternatively, all-optical systems can be used, and they have particular benefits, such as being insensitive to electromagnetic interference, not having the danger of initiating gas explosions with electric sparks, and allowing for high speed readout. Guo and co-workers employed an optical polymer microring resonator as an ultrasound sensor, in which the deformation of the resonator was measured by monitoring its shift in resonance frequencies [2,3]. Taillaert et al. [4] proposed the use of a silicon optical microring resonator as a strain gauge. Silicon-on-insulator (SOI) technology has emerged as a focus platform for integrated photonics, with complementary metal-oxide semiconductor (CMOS) production lines, opening the possibility of mass fabrication [5]. Silicon is the commonly used material in MEMS, and we have shown the possibility of micromachining postprocessing of SOI photonic integrated circuits [] $]$. Strong lateral confinement of the light due to the high refractive index contrast of SOI waveguides $(\Delta n \approx 2)$ allows for small device footprint, but also comes with high sensitivity to the exact behavior of the modes in the waveguide, e.g., strong modal dispersion $[7,8]$. Amemiya et al. [9] reported on the photoelastic effect in strained SOI ring resonators without, however, considering the modal effects, such as dispersion. In this Letter, we first derive a model explaining the effects that play a role when considering the influence of strain on photonic waveguides. Then, we characterize these effects with a novel mechanical setup providing a well-defined strain.

We describe the important effects when a strain is applied to a ring resonator that has a racetracklike shape with circumference $l$, as depicted in Fig. 1. Light is coupled from a connecting waveguide to the racetrack waveguide by means of a multimode interference (MMI) coupler $[10,11]$. Having such a long racetrack allows us to neglect the effect of the bends and of the coupler. The transmitted spectrum at the output port of the connecting waveguide shows dips at the wavelengths $\lambda_{m}$ in air of the resonances $m$ of the racetrack, given by

$$
m \cdot \lambda_{m}=n_{e}\left(\lambda_{m}, S\right) \cdot l(S) \text {. }
$$

The dependence of the effective index $n_{e}$ on wavelength, i.e., the modal dispersion, and on strain $S$ is explicitly shown. We apply a strain in the longitudinal direction of the racetrack due to which these resonance wavelengths shift. Three physical effects play a role when elongating the racetrack. First, the circumference of the racetrack is elongated. Second, the shape of the cross section of the waveguide is shrunk due to Poisson's effect. Third, the refractive index of the silicon itself is changed due to the photoelastic effect. The latter two effects influence together the effective index. For small strain $S$, the circumference $l=l_{0}(1+S)$, where $l_{0}$ is the circumference of the unstrained racetrack. Taking the partial derivative of Eq. (1) with respect to the strain $S$ gives

$$
m \frac{\partial \lambda_{m}}{\partial S}=\frac{\partial n_{e}}{\partial S} l+\frac{\partial n_{e}}{\partial \lambda_{m}} \frac{\partial \lambda_{m}}{\partial S} l+n_{e} \frac{\partial l}{\partial S},
$$

which we evaluate at zero strain $(S=0)$. Solving this equation for $\partial \lambda_{m} / \partial S$, substituting $m$ from Eq. (1) at zero

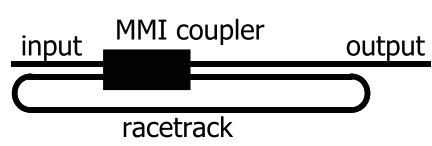

(a)

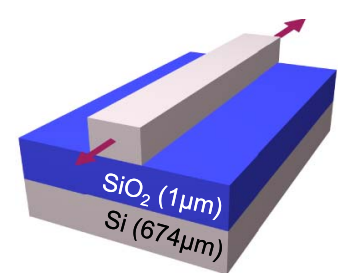

(b)
Fig. 1. (Color online) (a) Sketch of the racetrack resonator. (b) Sketch of the waveguide of which the racetrack consists, with elongation indicated by the arrows. 


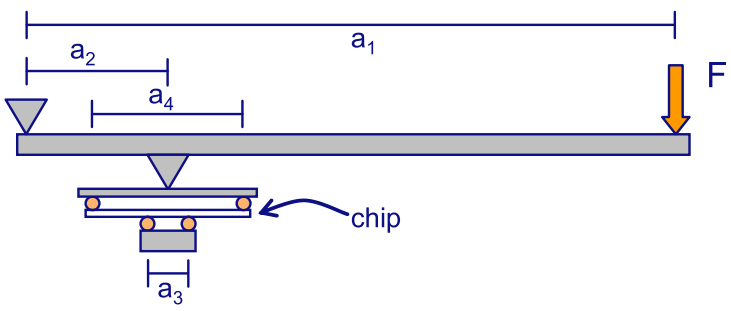

Fig. 2. (Color online) Mechanics of the tool for applying strain (schematic). The strain at the top surface of the chip is given by $S=6 a_{1}\left(a_{4}-a_{3}\right) F /\left(4 a_{2} E W H^{2}\right)$, where $F$ is the force, $E=170 \mathrm{GPa}$ is Young's modulus of $\mathrm{Si}$, and width $W=$ $20 \mathrm{~mm} \times$ height $H=0.675 \mathrm{~mm}$ are the dimensions of the chip. This relation is derived from a mechanical analysis of the setup, where Euler-Bernoulli beam bending theory is applied to describe the bending of the chip [12].

strain, and substituting $\partial l / \partial S=l_{0}$, gives the linearized influence of the strain:

$$
\frac{\partial \lambda_{m}}{\partial S}=\underbrace{\frac{n_{e}}{n_{g}}}_{\text {dispersion }}(\underbrace{\lambda_{0}}_{\text {circumference }}+\underbrace{\frac{\lambda_{0}}{n_{e}} \frac{\partial n_{e}}{\partial S}}_{\text {index }}),
$$

where the effective group index $n_{g} \equiv n_{e}-\lambda\left(\partial n_{e} / \partial \lambda\right)$. The influences of modal dispersion, change in circumference, and change in effective index on the wavelength shift are indicated.

In the remainder of this Letter, we characterize the different terms in Eq. (3) for typical SOI waveguides, by measuring the net wavelength shift, $\partial \lambda_{m} / \partial S$. The effective index $n_{e}$ and the group index $n_{g}$ are calculated with a mode solver (Film-Mode-Matching method in the FimmWave software by Photon Design Ltd, Oxford, UK). This allows us to calculate the influence of strain on the effective index of the waveguide, $\partial n_{e} / \partial S$.

For the strain measurements we designed a novel mechanical setup (Fig. 2) in which elastic bends are used to provide a uniform strain in the region between the two middle supports, where the microresonator is placed. Light was coupled to the silicon input and output waveguides by means of a lensed optical fiber that was positioned under an angle of $45 \mathrm{deg}$ with respect to the surface of the chip, resulting in a coupling loss of $\sim-20 \mathrm{~dB}$ per interface. The deflection of the chip is so small that the alignment maintains when strain is applied. We fabricated two long racetrack resonators (length $1040 \mu \mathrm{m}$, radius $25 \mu \mathrm{m}$ ), oriented in the [110] crystalline direction, in a $300 \mathrm{~nm}$ high silicon guiding layer. Waveguides with widths of 400 and $1000 \mathrm{~nm}$ where considered to explore the geometrical dependence of the effects. Broadband light with a free-space wavelength $\lambda_{0}$ around $1550 \mathrm{~nm}$ was used to excite the fundamental

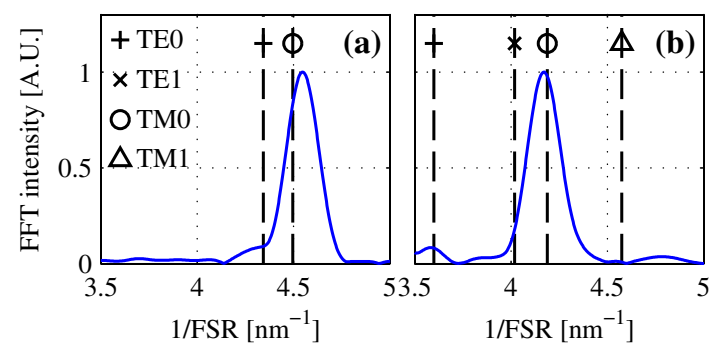

Fig. 3. (Color online) Fourier transforms of the spectra of the zero-strain measurements. The (FSR) ${ }^{-1}$ corresponding to the group indices $n_{g}$ as calculated with a mode solver are indicated. (a) $400 \mathrm{~nm}$ wide waveguide. (b) $1000 \mathrm{~nm}$ wide waveguide.
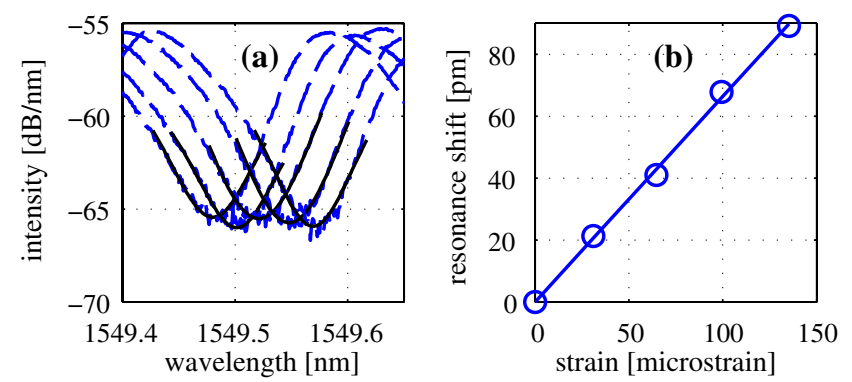

Fig. 4. (Color online) Measurement of resonance shift in the $1000 \mathrm{~nm}$ wide waveguide. (a) Five measured spectra with different strains. (b) Resonance wavelength of the dip of (a) plotted versus strain.
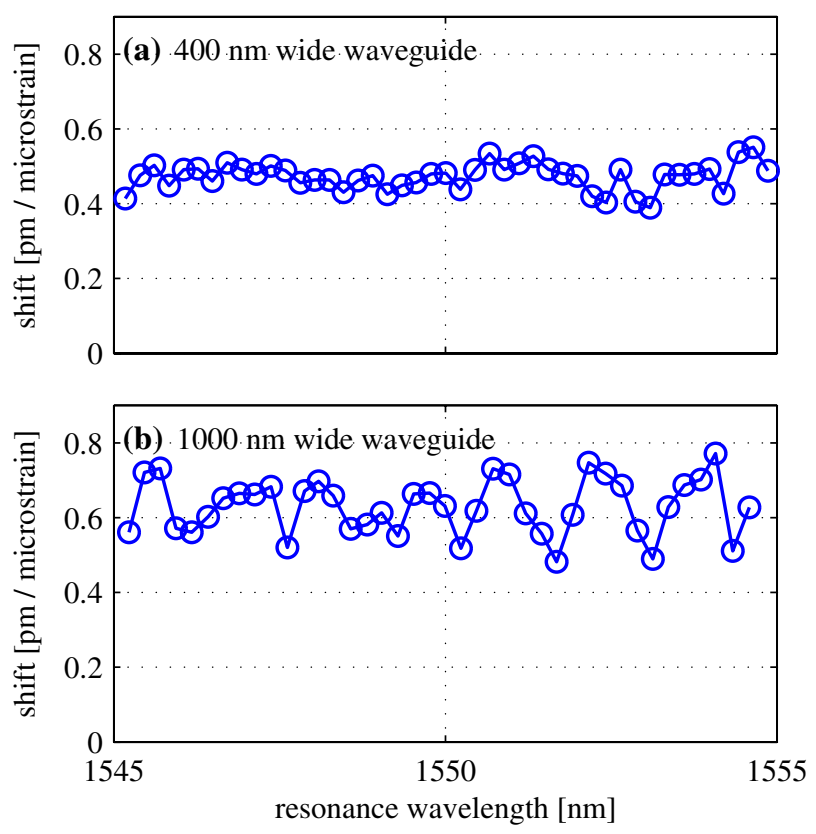

Fig. 5. (Color online) Measured shift of the resonance wavelengths due to applied strain.

Table 1. Effects of Strain

\begin{tabular}{cccccc}
\hline $\begin{array}{c}\text { Waveguide } \\
\text { Width [nm] }\end{array}$ & $\begin{array}{c}\text { Measured } \\
\text { Shift [pm/ } \mu \mathrm{\epsilon}]\end{array}$ & $\begin{array}{c}\text { Index } \\
\text { Change } \partial n_{e} / \partial S\end{array}$ & $\begin{array}{c}\text { Dispersion } \\
\text { Contribution }\end{array}$ & $\begin{array}{c}\text { Length } \\
\text { Contribution } \\
{[\mathrm{pm} / \mu \mathrm{\epsilon}]}\end{array}$ & $\begin{array}{c}\text { Index Contribution } \\
{[\mathrm{pm} / \mu \mathrm{\epsilon}]}\end{array}$ \\
\hline 400 & $0.47 \pm 0.04$ & $-0.73 \pm 0.13$ & 0.45 & 1.55 & -0.52 \\
1000 & $0.63 \pm 0.08$ & $-0.69 \pm 0.24$ & 0.56 & 1.55 & -0.39 \\
\hline
\end{tabular}


TM mode in the racetrack, which was verified by considering the Fourier transform of the measured spectrum. As the signal is periodic in the free-spectral range (FSR) of the resonator, ideally one intensity peak shows up at the reciprocal FSR, $(\mathrm{FSR})^{-1}=n_{g} \cdot l / \lambda_{0}^{2}$, which corresponds to the group index of a certain mode. In Fig. 3 it can be seen that the measurements agree excellently with the simulated group index of the fundamental TM-like mode in the waveguide. For each measurement, five transmission spectra are recorded with strain varying from 0 to 140 microstrain $(\mu \epsilon)$, as shown in Fig. 4(a). The position of the resonance dips are found by fitting Lorenzian distribution. Figure 4(b) shows the resonance wavelength versus strain, where $\partial \lambda_{m} / \partial S$ is obtained with a linear fit. Noise gives an error in the measured resonance wavelength [see Fig. 4(a)], therefore we take the mean shift of a large number of resonances and estimate the error as the standard deviation of the random dip-to-dip variation. The error in the effective indices is neglected, which is supported by good agreement of the simulated and measured group index, as shown in Fig. 3.

Figure 5 presents the strain-induced shifts of all the resonances of the racetrack. From these measurements, we calculate the different effects that play a role (also see Eq. 3), and present them in Table 1. Modal dispersion, i.e., the factor $n_{e} / n_{g}$, reduces the wavelength shift by a factor 2. The elongation of the track length increases the resonance wavelength, whereas the strain-induced effective index change reduces the resonance wavelength and, hence, the two effects oppose each other. The net wavelength change per unit strain caused by the effective index change is $1 / 3$ of the change caused by elongation, so that the net effect is a positive wavelength shift. Furthermore, we observe a weak dependence of the change in effective index, $\partial n_{e} / \partial S$, on the width of the waveguide.

In conclusion, we demonstrated that strain can be measured with integrated silicon optical microresona- tors. When considering the shift of the optical resonance wavelength, the following effects play an important role: elongation of the racetrack length, modal dispersion of the waveguide, and the strain-induced change in effective refractive index.

\section{References}

1. A. A. Barlian, W.-T. Park, J. R. Mallon Jr., A. J. Rastegar, and B. L. Pruitt, Proc. IEEE 97, 513 (2009).

2. C.-Y. Chao, S. Ashkenazi, S.-W. Huang, M. O'Donnell, and L. J. Guo, IEEE Trans. Ultrason. Ferroelectrics Freq. Contr. 54, 957 (2007).

3. T. Ling, S.-L. Chen, and L. J. Guo, Opt. Express 19, 861 (2011).

4. D. Taillaert, W. Van Paepegem, J. Vlekken, and R. Baets, Proc. SPIE 6619, 661914 (2007).

5. P. Dumon, W. Bogaerts, A. Tchelnokov, J.-M. Fedeli, and R. Baets, Future Fab International 25, 29 (2008).

6. J. Pozo, W. J. Westerveld, P. J. Harmsma, S. Yang, P. Bodis, R. Nieuwland, M. Lagioia, D. M. R. Lo Cascio, J.-P. Staats, R. Schmits, H. van den, Berg, E. Tabak, K. Green, H. P. Urbach, L. K. Cheng, and M. Yousefi, in Proceedings of the 13th International Conference on Transparent Optical Networks (ICTON) (National Institute of Telecommunications, 2011), paper Th.A4.5.

7. W. J. Westerveld, S. M. Leinders, K.W.A. van Dongen, H. P. Urbach, and M. Yousefi, "Extension of Marcatili's analytical approach for rectangular silicon optical waveguides," J. Lightwave Technol. (submitted).

8. P. Dumon, G. Priem, L. R. Nunes, W. Bogaerts, D. van Thourhout, P. Bientsman, T. K. Liang, M. Tsuchiya, P. Jaenen, S. Beckx, J. Wouters, and R. Baets, Jpn. J. Appl. Phys. 45, 6589 (2006).

9. Y. Amemiya, Y. Tanushi, T. Tokunaga, and S. Yokoyama, Jpn. J. Appl. Phys. 47, 2910 (2008).

10. A. Yariv, Electron. Lett. 36, 321 (2000).

11. L. B. Soldano and E. C. M. Pennings, J. Lightwave Technol. 13, 615 (1995).

12. J. M. Gere and S. P. Timoshenko, Mechanics of Materials (Stanley Thornes, 1999). 